\title{
Influence of Separation Interference Method on aerodynamic responses of a pentagonal shaped bridge deck
}

\author{
Md. Naimul Haque ${ }^{* * *}$ and Hiroshi Katsuchi** \\ *Dept. of Civil Eng. East West University, A/2, Aftabnagar, Dhaka-1212, Bangladesh \\ **Dept. of Civil Eng., Yokohama National University, Tokiwadai, Hodogaya-ku, Yokohama 240-8501, Japan \\ naimul@ewubd.edu (Corresponding Author)
}

\begin{abstract}
Long-span bridges often exhibit vibration under wind action. Various aerodynamic countermeasures have been developed over the years to enhance the aeroelastic responses of the long-span bridge deck and Separation Interference Method (SIM) is one of these. Engineers should have sound knowledge about the flow mechanism of the aerodynamic countermeasures of the bridge deck for further improvement of the aerodynamic responses and better design of the bridge deck. In the present study, a numerical investigation was carried out to disclose the flow mechanism of SIM on a pentagonal bridge deck. Simulation was conducted by using unsteady RANS for a pentagonal bridge deck with and without SIM techniques and their aerodynamic responses were compared. In the first part performance of unsteady RANS was evaluated to predict the dynamic responses for a pentagonal bridge deck. Then, the steady state responses and flow fields were explored for a bridge deck with and without SIM. Later, the dynamic responses such as flutter derivatives and unsteady pressure characteristics were evaluated and compared. It was found that SIM can improve the aerodynamic responses of the bridge deck. The addition of the curb affected the flow field around the bridge deck and the important flow features noticeably. The leading-edge top and bottom surface separations and the trailing-edge bottom surface separation are the most significant flow features to control both the steady state and dynamics responses of the bridge deck.
\end{abstract}

Keywords: Pentagonal bridge deck; Separation Interface Method; Unsteady RANS; Force coefficients; Flutter derivatives; aerodynamic damping

\section{INTRODUCTION}

Cable-stayed and suspension bridges are two common forms for long-span bridges. The deck of these bridges often exhibit instability against wind such as vortex-shedding vibration and flutter instability. Engineers adopt both aerodynamic and structural measures to improve the aerodynamic behavior and suppress the vibration. Aerodynamic countermeasures are more attractive to the engineers as it is less expensive and requires less maintenance compared to the structural countermeasures. Common aerodynamic countermeasures are fairing, flap and skirt etc. However, for each of these counter 
measures additional parts are required. It would be more promising if the aerodynamic responses of the bridge deck can be improved only by changing the size, shape or location of existing and essential bridge deck parts.

Kubo and his associates [1-3] proposed an aerodynamically stable deck which is pentagonal in shape as shown in Fig.1. To increase the aerodynamic stability of this kind of bridge deck no extra devices were required. Flow around the bridge deck can be modified by adjusting the slope of the bottom plate $(\theta)$ and position of the curb. Control and modification of flow by aligning the curb is familiar as separation interference method (SIM) [1,4]. The deck of the Takeshima Ohashi, Shintenmom, Oshima, Kesennuma and Ikara Ohashi bridges have adopted this concept to improve the stability of the deck against wind. For this kind of deck there are two important shaping features: i) the bottom plate slope $(\theta)$ and ii) the SIM (The presence of curb). The effect of bottom plate slope $(\theta)$ on aerodynamic responses such as the force coefficient, vortex shedding behavior and flutter instability was investigated by Kubo et al. [2]. Their investigation revealed that the pentagonal bridge with a bottom plate slope $(\theta)$ of $12^{\circ}$ experiences the highest flutter wind speed and smallest aerodynamic loading (specifically, the drag had the smallest value). On the other hand, the influence and effectiveness of SIM was mainly demonstrated for a square cylinder by Kubo et al. [4]. They showed that SIM can effectively reduce the magnitude of force coefficients and vibration of a square cylinder. It was the small vortex that develops at the windward side of the vertical plate what reduces the drag force and prevents the enrollment of periodic separated flow at the wake of the cylinder to reduce vibration. Based on that concept the curb was attached to a pentagonal bridge deck to take the advantage of SIM. However, the flow mechanism of SIM in the case of an elongated body like a pentagonal cylinder, where the separated flow reattaches at the top surface of the deck without enrolling directly at the wake of the body will differ from a square cylinder. No evidence of work regarding this issue was noticed in the literature. Therefore, it is important to reveal the flow mechanism of SIM for elongated bluff bodies like the bridge deck section.

Moreover, the flow field for this type of bridge was also analyzed at some level in previous works. The leading-edge top surface flow separation, leading-edge bottom surface flow separation and the trailing-edge bottom surface flow separation are the most important flow features for the bridge deck of this kind [5]. The role of these important flow features in controlling and modifying the steady state and dynamic response of a pentagonal bridge deck under wind action has not been fully revealed yet. Noda et al. [3] revealed a few implications of these flow features for steady state force coefficients. 
They showed that the leading-edge bottom surface separation has significant correlation with the steady state lift force coefficient. Later, Haque et al. [5] devoted a numerical investigation where they partially disclosed the function of these important flow features in controlling and modifying the steady state aerodynamic responses. They also clarified the effect of the slope of bottom plate $(\theta)$ and Reynolds number $\left(R_{e}\right)$ on these important flow features. They found that the leading-edge flow features have influences on the mean values of steady state force coefficient and the trailing edge flow separation has influence on rms values of steady state force coefficient. They also revealed that all these flow features are sensitive to the shape of the deck and Reynolds number $\left(R_{e}\right)$. Past studies could partially reveal the role of those flow features on steady state responses only. However, there is still a lot to reveal about the flow mechanism of this kind of bridge deck, specially the role of these flow features on dynamic responses.

Further, the applicability of Computational Fluid Dynamics (CFD) is increasing gradually in bridge aerodynamic field to reconfirm the past experimental works and gain confidence form newly proposed technique. Researchers [6-9] are currently employing CFD to understand better way the aerodynamic responses and explore the mechanism behind. Therefore, it is important to check the efficiency of SIM by numerical methods like CFD to enhance the understanding about the aerodynamics of pentagonal bridge deck and strengthen the previous observations. Large Eddy Simulation (LES) and unsteady Reynolds-Averaged Navier-Stokes (RANS) are two common approaches in CFD adopted by the researchers. As the boundary layer length of bridge deck is much longer as compared to the other bluff bodies, researchers mostly adopted unsteady RANS in bridge aerodynamics field due to it is computationally inexpensive nature.

With this background, in the present study a series of simulations were conducted to show the influence of SIM on aerodynamic response of a pentagonal bridge deck and the flow mechanism behind that. The objective of the present study is to reveal the role of various important flow features on dynamic response of a pentagonal shaped bridge deck. In the first part of the paper, the performance of unsteady RANS was evaluated by calculating and comparing the aerodynamic responses of the pentagonal bridge deck with the wind tunnel data. Then, numerical computations were conducted for a pentagonal bridge deck with and without curb to observe the influence of SIM on static and dynamic responses under wind and the flow field was analyzed to understand the role of various flow features on aerodynamic responses. Fig.2 shows the considered bridge deck. Various aerodynamics parameters such as the mean and rms value of steady state force and 
pressure coefficients were evaluated to quantify the effect of SIM. Finally, the flutter derivatives and aerodynamic damping of the considered bridge deck were evaluated and compared to illustrate the effectiveness of SIM and understand the role of the important flow features on dynamic responses.

\section{NUMERICAL METHOD}

The ensemble averaged Navier-Stokes equations known as unsteady Reynolds-Averaged Navier-Stokes (URANS) equation were solved to simulate the flow. The governing equations are listed below;

$$
\begin{gathered}
\frac{\partial \bar{U}_{i}}{\partial x_{i}}=0 \\
\frac{\partial \bar{U}_{i}}{\partial t}+\bar{U}_{j} \frac{\partial \bar{U}_{i}}{\partial x_{j}}=-\frac{1}{\rho} \frac{\partial \bar{P}}{\partial x_{i}}+\frac{\partial}{\partial x_{j}}\left[\mu\left(\frac{\partial \bar{U}_{i}}{\partial x_{j}}+\frac{\partial \bar{U}_{j}}{\partial x_{i}}\right)-\left(\overline{u_{j}^{\prime} u_{i}^{\prime}}\right)\right]
\end{gathered}
$$

where, $x_{i}$ and $\bar{U}_{i}$ are averaged position and velocity vectors respectively, $\bar{P}$ is the averaged pressure, $t$ is time, $\mu$ is the molecular viscosity and $\rho$ is air density. The Reynolds stress $\left(\rho \overline{u_{j}^{\prime} u_{i}^{\prime}}\right)$ was modelled by $k$ - $\omega$-SST [10-11] turbulence model. In $k$ - $\omega$-SST model the Reynolds stress $\left(\rho \overline{u_{j}^{\prime} u_{i}^{\prime}}\right)$ was modelled based on the Boussinesq hypothesis which is written as follows,

$$
-\rho \overline{u_{j}^{\prime} u_{i}^{\prime}}=2 \mu_{t} S_{i j}-\frac{2}{3} \rho k \delta_{i j}
$$

where, $\mu_{t}$ is the eddy viscosity and $S_{i j}$ is the strain rate of tensor. To discretize the governing equations, Finite Volume Method (FVM) was utilized. The domain size is shown in Fig.3. At the bridge deck surface, a non-slip type boundary condition $(\partial u / \partial y \neq 0$ and $v=0)$ was enforced. At the domain inlet, for velocity $(u=U$ and $v=0)$ a dirichlet type boundary condition and for pressure $(\partial p / \partial n=0)$ Neumann type boundary condition were implemented. On the other hand, at the domain outlet Neumann and dirichlet type boundary condition were applied for the velocity and pressure respectively. At the domain top and bottom surface, a slip type boundary condition $(\partial u / \partial y=0$ and $v=0)$ was applied. Further details of the numerical schemes used to discretize the governing equations and justification of the considered domain size can be found in Haque et al. [5,12]. OpenFOAM was used as a solver in the present investigation. 
To discretize the domain spatially a body fitted grid system was chosen where the cell size was varied gradually. The average $y^{+}$value was maintained below 5 and the first cell height $(y)$ near the bridge deck was selected accordingly. At present Reynolds number $\left(R_{e}=6.0 \times 10^{4}\right)$ the average $y^{+}$value was near about 2 and the value of maximum $y^{+}$reached up to 8.2. At the remaining area of the bridge deck the $y^{+}$value was well below 5 . To obtain an accurate result it should be ensured that there are a sufficient number of grids and results are converged. In previous study [5] detailed convergence test was conducted for the considered pentagonal bridge deck at the present Reynolds number $\left(R_{e}=6.0 \mathrm{x} 10^{4}\right)$. Therefore, the same grid system was chosen in the present study as described above. The Courant number $\left(C_{o}\right)$ was utilized to select the required time step size $\left(\Delta t=\left(C_{\delta} \Delta x\right) / U\right.$, where, $\Delta x$, was the smallest grid size $)$ for temporal discretization. The maximum Courant number $\left(C_{o}\right)$ was maintained at about 0.5 .

In the current study, along with the static simulation, forced vibration simulation was also carried out to predict the flutter derivatives and unsteady pressure characteristics. Therefore, the governing equations were modified as follows:

$$
\begin{gathered}
\frac{\partial\left(\bar{U}_{i}-\bar{U}_{g i}\right)}{\partial x_{i}}=0 \\
\frac{\partial \bar{U}_{i}}{\partial t}+\bar{U}_{j} \frac{\partial\left(\bar{U}_{i}-\bar{U}_{g i}\right)}{\partial x_{j}}=-\frac{1}{\rho} \frac{\partial \bar{P}}{\partial x_{i}}+\frac{\partial}{\partial x_{j}}\left[v\left(\frac{\partial \bar{U}_{i}}{\partial x_{j}}+\frac{\partial \bar{U}_{j}}{\partial x_{i}}\right)-\left(\overline{u_{i}^{\prime} u_{j}^{\prime}}\right)\right]
\end{gathered}
$$

where $\bar{U}_{g i}$ is the grid velocity in the $i$-th direction.

To conduct forced vibration simulation the boundary of the bridge deck was moved periodically by deforming the grid around the bridge deck. Arbitrary Lagrangian Eulerian (ALE) [13-14] method was used to move and deform the computational grid by following the movement of the boundary in every time step of transient simulation. An interpolation based Radial Basis Function (RBF) [15] was used to calculate the motion of the internal points. In the case of dynamic simulation the pressure-velocity coupling was attained by PIMPLE algorithm. The maximum courant number $\left(C_{o}\right)$ was kept well below a value of 1 to ensure stability of the simulations.

\section{DEFINITION OF AERODYNAMIC FORCES}

In the present analysis, the steady state force coefficients were calculated by using the following equations, 


$$
\begin{aligned}
& C_{D}=\frac{F_{D}}{1 / 2 \rho U^{2} D} \quad \text { (Downstream positive) } \\
& C_{L}=\frac{F_{L}}{1 / 2 \rho U^{2} B} \quad \text { (Upward positive) } \\
& C_{M}=\frac{F_{M}}{1 / 2 \rho U^{2} B^{2}} \quad \text { (Anti-clockwise positive) } \\
& S_{t}=\frac{f D}{U}
\end{aligned}
$$

where $C_{D}$ is the drag force coefficient, $C_{L}$ is the lift force coefficient, $C_{M}$ is the moment coefficient, $S_{t}$ is the Strouhal number, $F_{D}$ is the drag force, $F_{L}$ is the lift force, $F_{M}$ is the moment force and $f$ is the frequency of the vortex shedding. It was reported by Kubo et al. [2] and Noda et al. [3] that the pentagonal bridge deck exhibits torsional flutter. Therefore, in the present study only torsional flutter derivatives were evaluated. In forced vibration simulation the aerodynamic lift and moment forces are expressed by the following expressions as mentioned in Simiu and Scanlan [16]:

$$
\begin{aligned}
& L(t)=\frac{1}{2} \cdot \rho \cdot U^{2} \cdot B \cdot\left[k \cdot H_{2}^{*} \cdot B \cdot \frac{\dot{\alpha}}{U}+k^{2} \cdot H_{3}^{*} \cdot \alpha\right] \\
& M(t)=\frac{1}{2} \cdot \rho \cdot U^{2} \cdot B^{2} \cdot\left[k \cdot A_{2}^{*} \cdot B \cdot \frac{\dot{\alpha}}{U}+k^{2} \cdot A_{3}^{*} \cdot \alpha\right]
\end{aligned}
$$

where $L(t)$ is the self-excited time-varying lift force and $M(t)$ is the self-excited time-varying moment per unit span of the bridge deck; $\rho$ is the density of air; $k$ is reduced frequency, $(k=B . \omega / U) ; U$ is the mean wind speed; $\omega$ is circular frequency, $\left(2 . \pi . f_{\alpha}\right) ; B$ is the bridge deck full width; $\alpha$ is the torsional displacement; the dot (') represents first time derivatives; and the $H_{i}^{*}$ and $A_{i}^{*}$ are the aerodynamic coefficients known as flutter derivatives in heaving and torsional motions, respectively. The same procedure of extracting the flutter derivatives was utilized as discussed (elaborately) in past works [9, 17-21]. By changing the frequency of rotation $\left(f_{\alpha}\right)$ the reduced velocity $\left(U / f_{\alpha} B\right)$ was altered.

Flutter derivatives, which provide the general information about the genesis of flutter instability can be obtained. However, for detailed understanding of aerodynamic instability, the unsteady pressure distribution should be considered, which provide meaningful information about the complex fluid-structure interaction. The torsional oscillation was defined by 
cosine function and the total pressure coefficient $\left(C_{p}(x, t)\right)$ was defined as follows: .

$$
\begin{aligned}
& \alpha(t)=\alpha_{o} \cdot \operatorname{Cos}\left(2 \cdot \pi \cdot f_{\alpha} \cdot t\right) \\
& C_{p}(x, t)=\bar{C}_{p}(x)+C_{p}{ }^{\prime}(x, t)
\end{aligned}
$$

where, $\alpha(t)$ is the torsional displacement at a given time $t, C_{p}(x, t)$ is the total pressure coefficient, $\alpha_{o}$ is the amplitude of torsional oscillation, $f_{\alpha}$ is the torsional oscillation frequency of the model, $\bar{C}_{p}(x)$ is the mean pressure coefficient, and $C_{p}{ }^{\prime}(x, t)$ is the unsteady pressure coefficient. The unsteady pressure coefficient $\left(C_{p}{ }^{\prime}(x, t)\right)$ was defined by the amplitude of unsteady pressure $\left(\left|C_{p}(x)\right|\right)$ and the phase $\operatorname{leg}(\varphi(x))$ among the pressure and the motion of the bridge deck as shown below.

$$
C_{p}{ }^{\prime}(x, t)=-\left|C_{p}(x)\right| \cdot \operatorname{Cos}(2 \cdot \pi \cdot f \cdot t+\varphi) .
$$

The magnitude of unsteady pressure $\left(\left|C_{p}(x)\right|\right)$ was taken as the amplitude of the periodic pressure coefficient history caused by the forced vibration only and ignoring the high frequency component due to vortex shedding. The phase lag $(\varphi(x))$ was defined as a difference in phase among the highest angle of attack of the bridge deck and the highest negative pressure at a distance $x$.

The imaginary part of the unsteady pressure $\left(C_{p}{ }^{\prime}(x, t)\right)$ is defined as, $C_{P I}=\left|C_{p}(x)\right| \cdot \operatorname{Sin} \varphi(x)$ is associated to the aerodynamic damping of the bridge deck [22-25]. In the present work for torsional motion, the positive value was considered as an excitation force and negative value was a damping force on the leading edge side top surface of the deck and was altered at the trailing edge side top surface of the bridge deck. Opposite sign convention was adopted on the bottom surface in relation to the top surface of the deck at the leading and trailing edge side. The work done by the unsteady pressure can be calculated as follows,

$$
C_{P I} \cdot r=(x / B) \cdot\left|C_{P}(x)\right| \cdot \operatorname{Sin} \varphi(x)
$$

where $r$ is the normalized distance from the center of the bridge deck to the both sides.

\section{CAPABILITY OF UNSTEADY RANS}

Capability of Unsteady RANS has already been checked for predicting steady state and dynamic responses in a number 
of works. However, depending on the shape of the target body, the performance of predicting aerodynamic responses varies as the nature of flow around the body is different for different bodies. Researchers are still taking opportunities to validate the results of this numerical method for new objects and responses which have not yet been validated to widen the application range. Validation of the numerical results is also important so that the cons and pros of the method can be discovered to explain the obtained numerical results on a better way.

In the bridge aerodynamic field, the performance of unsteady RANS has mainly been evaluated for streamlined bridge decks. Bruno and Mancini [6] conducted steady state numerical simulation by using unsteady RANS for the Normandy and Great Belt East bridge and compared the pressure coefficients with wind tunnel data. Mannini et al. [7] also compared steady state force and pressure coefficients of a single-box girder deck with a lateral cantilever. Some other researchers devoted towards the dynamic responses of the bridge deck such as flutter derivatives. Shirai and Ueda [26] conducted a series of simulations for a slotted bridge deck and compared the results with past wind tunnel data. Šarkić et al. [18] and Brusiani et al. [19] conducted forced vibration simulation for a streamlined bridge deck and compared the flutter derivatives with the experimental ones. Patruno [9] choose a number of practically constructed bridges and compared the numerically calculated flutter derivates with the experimental data.

The bridge deck considered in the present analysis is different from previously considered bridge decks. The deck is bluff in shape and possesses as sharp edges. It would be interesting to check the capability of unsteady RANS for the considered bridge deck. The steady state responses for the current pentagonal shaped bridge deck are already verified and validated in Haque et al. [5]. In Haque et al. [5], a detailed verification process was launched which validated both the pressure coefficients and velocity fields. Reasonable accordance was observed between the numerical and experimental results.

In the current investigation, the same numerical setup, grid size and Reynolds number were considered. As the steady state responses are already validated previously, those results are not presented here. The target of this section would be to validate the dynamic responses such as the flutter derivatives and unsteady pressure characteristics for the current bridge deck in torsional mode as this kind of bridge deck exhibits only torsional mode of flutter [2-3]. Matsumoto et al. [27] reported two torsional mode flutter derivatives $\left(A_{2} *\right.$ and $H_{3} *$ for the inverse-triangle section $\left(R=6\right.$ and $\left.\left.\theta_{B}=13.33^{\circ}\right)\right)$ which is 
similar to the bridge deck without curb (Fig.2). Therefore, we calculated the torsional mode flutter derivatives for the same shape and compared the results with the wind tunnel data to validate the current numerical setup and check the performance of unsteady RANS.

Fig. 4 shows the grid system utilized to discretize the domain for the pentagonal bridge deck without curb. The Reynolds number $\left(R_{e B}\right)$ was set to $6.0 \times 10^{4}$ and forced torsional vibration simulation was carried out with a torsional amplitude $\left(\alpha_{o}\right)$ of $2^{\circ}$ to maintain similarities with the experimental test. Fig.5 illustrates the computed flutter derivatives along with the experimental results [27]. A reasonable accordance can be noticed between past experimental and present numerical result. It can be seen that $A_{2}{ }^{*}$ has a higher discrepancy compared to the $H_{3} *$ Similar observation was also reported in previous numerical investigations $[9,20,28]$ of those who attempted to validate rectangular cylinders and bridge decks of various shapes that $A_{2}{ }^{*}$ has little bit high discrepancy. However, in both of the cases in Fig.5, the unsteady RANS could grasp the trend in the results i.e. the increasing and decreasing nature of the flutter derivatives in relation to the reduced velocity and the sign.

Along with the flutter derivatives, another target response was the unsteady pressure and phase lag to calculate the work done by the unsteady pressure. However, in the literature there is not much data available regarding the unsteady pressure $\left(\left(\left|C_{P}\right|\right)\right.$ and the phase lag $(\varphi)$ of the bridge deck or bluff body. Matsumoto [29] reported the amplitude of unsteady surface pressure $\left(\left(\mid C_{P}\right)\right.$ and the phase lag $(\varphi)$ distribution for the elongated cylinder $(R=5)$ in the torsional mode of vibration. The rectangular cylinder and the target bridge deck (pentagonal shape) have similarities in terms of sharp corner and bluff in nature. Therefore, we decided to simulate the unsteady pressure amplitude $\left(\left(\left|C_{P}\right|\right)\right.$ and phase lag $(\varphi)$ of a rectangular cylinder $(R=5)$ and compare with the experimental results of Matsumoto [29]. Fig.6 shows the computational grid system for the rectangular cylinder. Fig.7 reports the amplitude of unsteady pressure $\left(\left(\left|C_{P}\right|\right)\right.$ and the phase lag $(\varphi)$ distribution for the rectangular cylinder $(R=5)$. As can be seen the unsteady RANS and the present numerical setup efficiently predict both the magnitude and trend in the results. We proceeded with this level of accuracy as relative comparison of aerodynamic responses will be made among the bridge deck of similar shapes.

\section{STEADY STATE RESPONSES}


The mean and RMS values of the steady state force coefficients are compared in Table 1 . As can be seen the presence of SIM on the bridge deck affects the force coefficients. Other than the drag force coefficients $\left(C_{D}\right)$ and Strouhal number $\left(S_{t B}\right)$ all the remaining force coefficients alters noticeably. In particular, the mean and rms values of lift force coefficients $\left(C_{L}\right)$ alter significantly. Drag reduction is one of the important issues in engineering. The attachment of the curb reduces the drag around $9 \%$. The mean absolute value of lift force coefficient increases and moment force coefficient decreases, however, the rms value of lift force coefficient increases. The pressure and velocity fields are explored elaborately in the following segment to understand how the presence of curb altered the force coefficient responses.

The mean value of pressure distribution around the considered deck sections is plotted in Fig.8. As can be seen the presence of the curb mainly affects the top pressure of the deck as compared to the bottom surface of the deck. The suction at the top surface drops noticeably due to the addition of the curb. This reduction of the suction decreases the negative value of the lift force coefficient $\left(C_{L}\right)$. Along with the top surface, the leading-edge bottom surface pressure of the deck is also affected by the presence of the curb. Contrary to the top surface pressure, the magnitude of suction increases here. Due to this increase in leading-edge suction, the negative lift and the positive moment values increase as reflected in Table 1 . The presence of the curb doesn't affect the bottom surface trailing-edge pressure distribution. The wind speed ratio around the bridge decks are compared in Fig.9. The velocity distribution around the bridge deck was normalized by the inlet wind speed. At the top surface leading-edge side of the deck, as can be seen that the flow separation decreased significantly due to attachment of the curb, while at the leading-edge bottom surface of the deck the flow separation increases. No significant variation can be seen at the wake of the bridge deck due to addition of curb. However, the bridge deck with curb has a slightly larger wake compared to the bridge deck without curb. The velocity distribution at the trailing edge of the bridge deck is plotted in the Fig.10. The bridge deck with curb has a slightly wider wake and larger boundary layer as compared to the bridge deck without curb. As a result, the bridge deck with curb has a higher drag value. The RMS value of the pressure distribution is compared in Fig.11. The bridge deck with curb has larger RMS value and the RMS value increases at the top surface trailing-edge side of the bridge deck. However, at the bottom surface, the RMS value increases throughout the length. It is difficult to presume whether this increase in RMS value will deteriorate the dynamic responses of the bridge deck against wind or not. Therefore, it is important to predict the dynamic responses of the bridge decks against wind. In the 
following section the flutter derivatives and unsteady pressure characteristics of the bridge decks are evaluated and compared.

\section{DYNAMIC RESPONSES}

Only torsional mode flutter derivatives of the bridge decks are considered for the present study as pentagonal bridge deck exhibits torsional flutter [2-3]. Forced vibration simulations were carried out with a torsional amplitude $\left(\alpha_{0}\right)$ of $1^{\circ}$. Fig.12 summarizes the calculated flutter derivatives of the considered bridge decks. $A_{2}{ }^{*}$ and $H_{2}{ }^{*}$ are the torsional and heaving mode aerodynamic damping during the time of torsional vibration. $A_{3}{ }^{*}$ and $H_{3}{ }^{*}$ are the torsional and heaving mode stiffness when the bridge deck is under torsional vibration. Fig.12 depicts that except $A_{2}{ }^{*}$, the other two flutter derivatives rarely alter due to addition of curb to the bridge deck. In the case of with curb situation, the coupled derivative $H_{3}{ }^{*}$ has smaller value which indicates better stability than the without curb case. However, the most important criteria would be the sign and magnitude of $A_{2}{ }^{*}$. As can be seen when there is no curb attached to the bridge deck, it shows an almost positive value for the complete range of reduced velocity. However, the magnitude of $A_{2}{ }^{*}$ becomes negative and the value increases with the increase in reduced velocity when the curb is attached to the section. This implies that the SIM improves the torsional flutter stability of the bridge decks under wind actions.

To better understand the influence of curb on dynamic responses, the work done by the unsteady pressure around the bridge deck is plotted in Fig.13. The excitation mainly exists at the leading-edge top and bottom surface of deck and damping force exists at the trailing edge side at low reduced velocity $(U / f B=5.05)$ for the considered bridge deck. Due to attachment of the curb, the excitation force increases a bit at the leading-edge top corner, yet drops significantly just after the curb. Both at the top and bottom surface trailing-edge side of the deck the aerodynamic damping increases due to addition of the curb. The effectiveness of SIM is evident at higher reduced velocity $(U / f B=15)$. The presence of the curb mainly affects the leading-edge side of the deck. At the leading-edge, the excitation force decreases at the top surface and bottom surface damping increases noticeably due to the addition of the curb.

In the last sections, we discussed the behavior of flow fields around the decks. There were a number of important flow features those are the leading-edge top surface flow separation and pressure recovery zone, leading-edge bottom surface 
flow separation and the trailing-edge bottom surface flow separation. The orientation and behavior of these flow features should be interconnected with the steady state and dynamic responses we discussed earlier. In the previous section, we discussed the probable role of those flow features on steady state responses. It is also important to disclose the function of these important flow features on dynamic responses. If a comparison is made between Fig. 9 and 13, the role of these flow features can be understood. The separated flow at the leading-edge top and bottom surface of the deck work as an excitation force, both at low $(U / f B=5.05)$ and high $(U / f B=15)$ reduced velocity, yet the magnitude of excitation decreases at high reduced velocity $(U / f B=15)$ as the intensity of flow separation lessens at high reduced velocity $(U / f B=15)[5]$. The pressure recovery zone at the trailing-edge of top surface works as a damping force at low reduced velocity $(U / f B=5.05)$ and becomes mostly excitation force at the high reduced velocity $(U / f B=15)$. Similar to the top surface trailing-edge pressure recovery zone, the bottom surface trailing-edge separated flow also works as a damping force at low reduced velocity $(U / f B=5.05)$, however, at high reduced velocity $(U / f B=15)$ it slightly becomes excitation force.

\section{CONCLUSIONS}

In the present study, unsteady RANS simulations were conducted for a pentagonal bridge deck with and without curb (SIM). The objective was to confirm the reliability of SIM on improving both steady state and dynamic responses against wind for a sharp edged elongated pentagonal bridge deck. Unsteady RANS simulation was employed to predict both the steady state and dynamic responses. The pressure distributions, flow fields and dynamic responses were explored in detail to reveal the role of various important flow features on aerodynamic responses. It was also intended to evaluate the performance of unsteady RANS for predicting the dynamic responses such as flutter derivatives and unsteady pressure characteristics of sharped edged bluff sections.

It was found that unsteady RANS can grasp the flutter derivatives and unsteady pressure characteristics with reasonable accuracy. In all cases the unsteady RANS could predict the trend in the results quite accurately. Discrepancy was noticed in between the experimental and numerical values of torsional flutter derivatives for predicting $A_{2}{ }^{*}$. However, the intensity of discrepancy was less in case of unsteady pressure amplitude and phase lag as compared to the flutter derivatives.

We also found that SIM is an effective aerodynamic countermeasure for a pentagonal bridge deck and can improve the 
aerodynamic responses. The incorporation of SIM improves the characteristics of force coefficients and flow fields around the bridge deck. The susceptibility of the bride deck against torsional flutter diminishes and the bridge deck becomes stable against torsional flutter due to the addition of curb. The flow separation decreases at the leading-edge top surface and the flow reattaches at the middle portion of the deck top surface when the curb is attached. The reduction of this flow separation reduces the upward lifting force and excitation force during the torsional vibration. However, the bottom surface leading and trailing-edge flow separation enhances with the inclusion of curb. The increase in leading-edge bottom surface separation enhances the downward lift force and the damping force during torsional vibration. The increase in trailing-edge bottom surface separation takes part into increase the drug force and aerodynamic damping of the bridge deck. However, before wide application of pentagonal bridge deck with SIM for medium to long-span bridges, further detailed investigation is required by taking into care of issues like turbulence and angle of attack of the inlet flow.

Table 1. Influence of SIM on steady state force coefficients

\begin{tabular}{lccc}
\hline Force coefficients & $\begin{array}{c}\text { Pentagonal bridge } \\
\text { deck without curb }\end{array}$ & $\begin{array}{c}\text { Pentagonal bridge } \\
\text { deck with curb }\end{array}$ & $\begin{array}{c}\text { Percentage } \\
\text { variation (\%) }\end{array}$ \\
\hline Mean value of drag force coefficient $\left(C_{D}\right)$ & 0.522 & 0.476 & -8.81 \\
Mean value of lift force coefficient $\left(C_{L}\right)$ & -0.032 & -0.356 & $>-100.00$ \\
Mean value of moment coefficient $\left(C_{M}\right)$ & -0.067 & -0.042 & +37.31 \\
rms value of lift force coefficient $\left(C_{L}\right)$ & 0.008 & 0.012 & +50.00 \\
rms value of moment coefficient $\left(C_{M}\right)$ & 0.002 & 0.002 & 0.0 \\
Strouhal number $\left(S_{B B}\right)$ & 0.897 & 0.855 & -4.68 \\
\hline
\end{tabular}

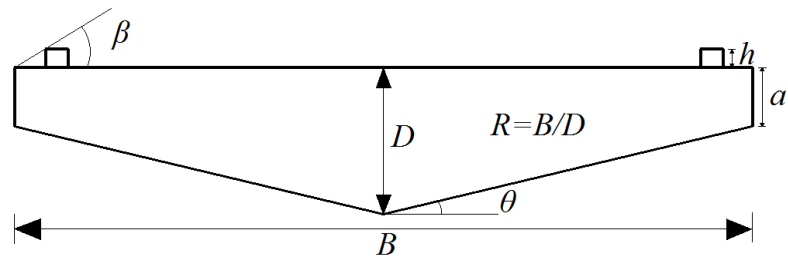

Fig. 1. Geometric configuration of the bridge deck which is stable aerodynamically 


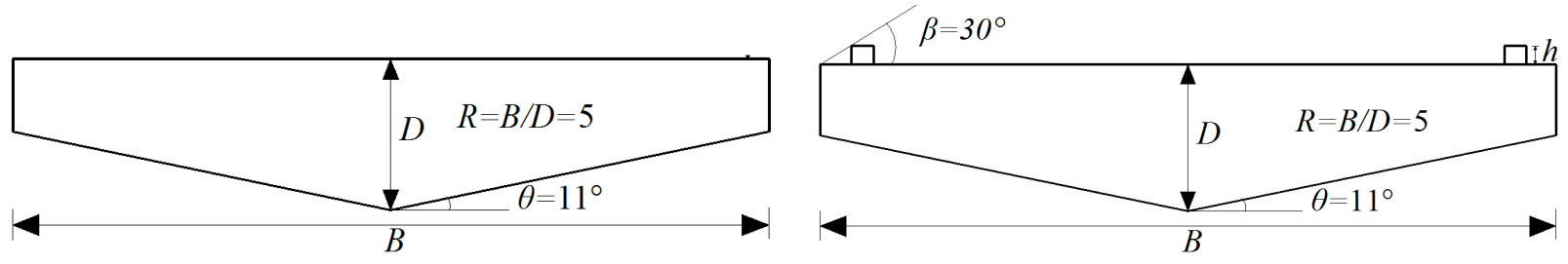

Fig. 2. Cross-section of the considered bridge $\operatorname{deck}(h / B=0.025)$

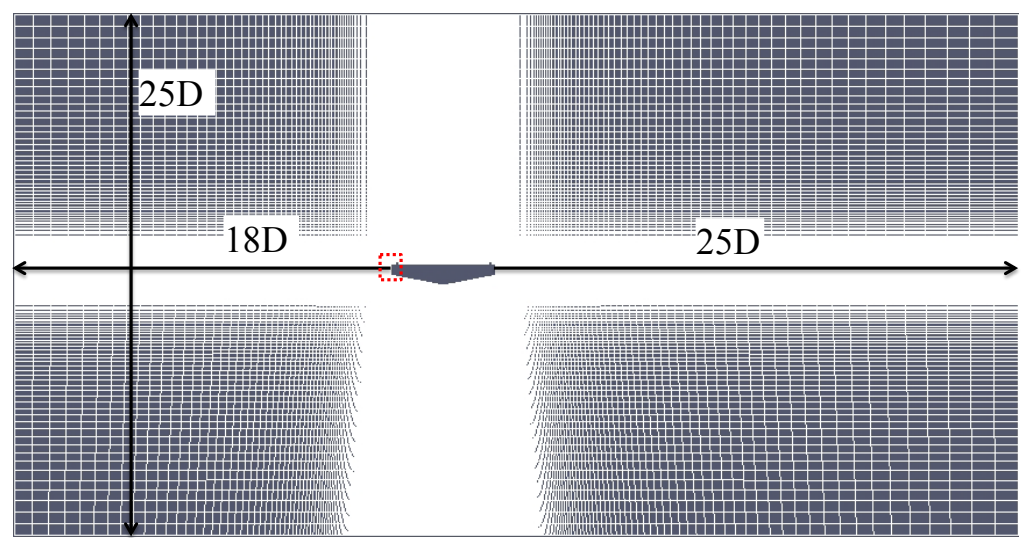

(a)

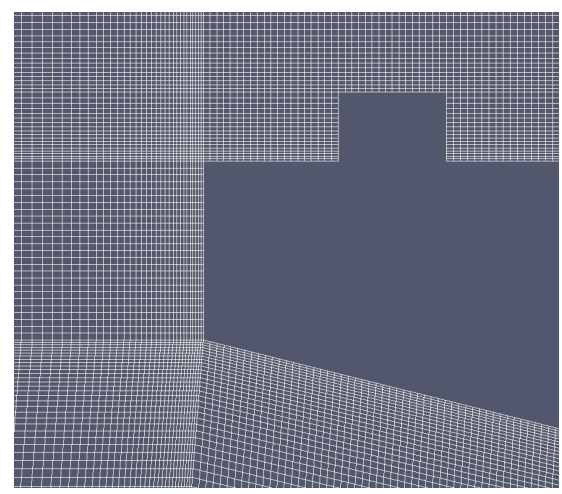

(b)

Fig. 3 Size of the domain and grid system ( $D$ is the bridge deck depth) adopted in the analysis: (a) Complete domain and (b) close-up look around the curb. Wind is blowing from left to right. 

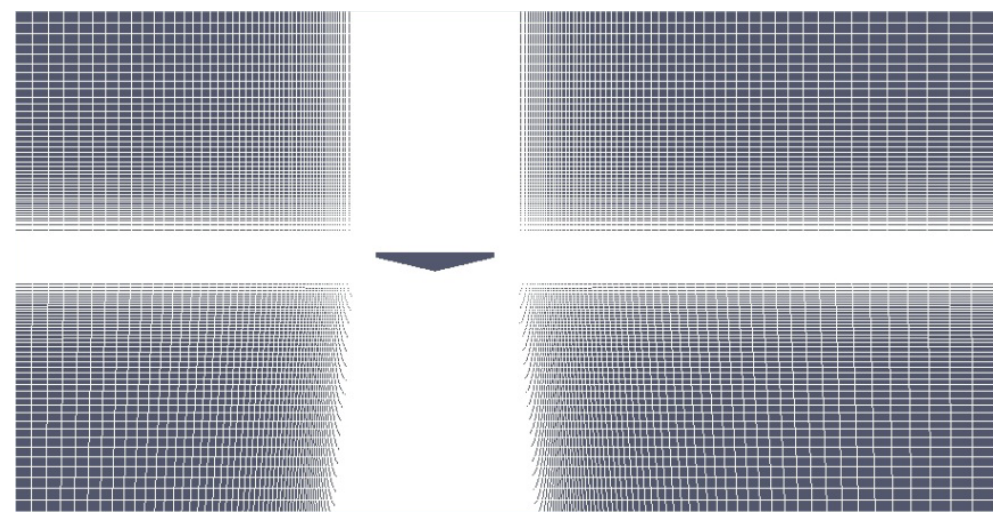

(a) The whole domain grid

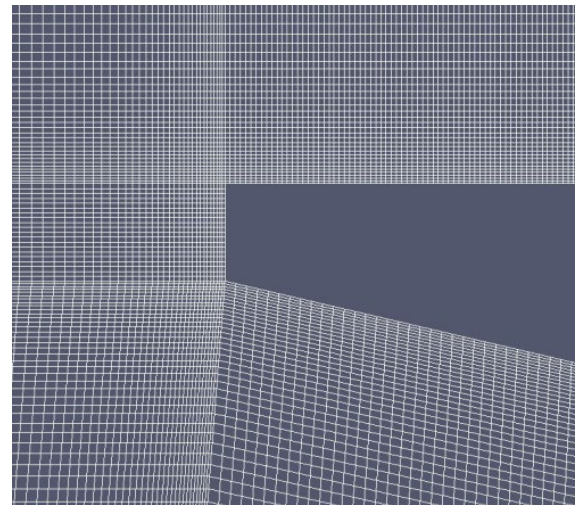

(b) Grid near the body

Fig. 4. Meshing of the pentagonal shaped bridge deck without curb

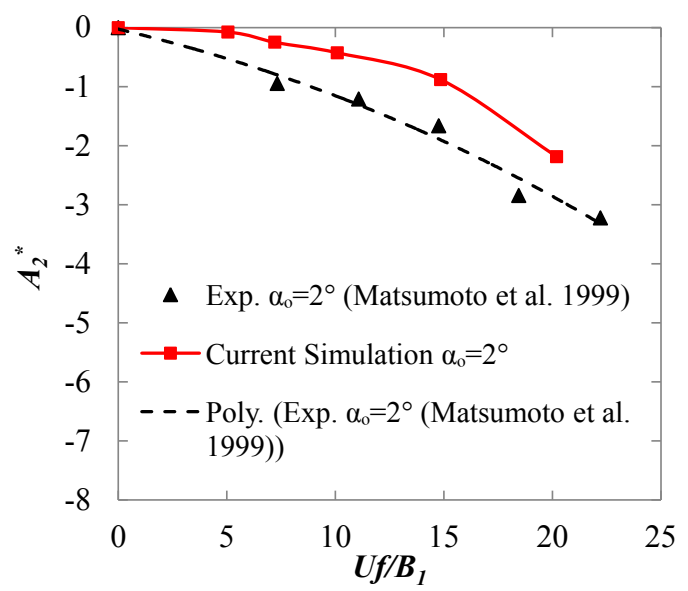

(a)

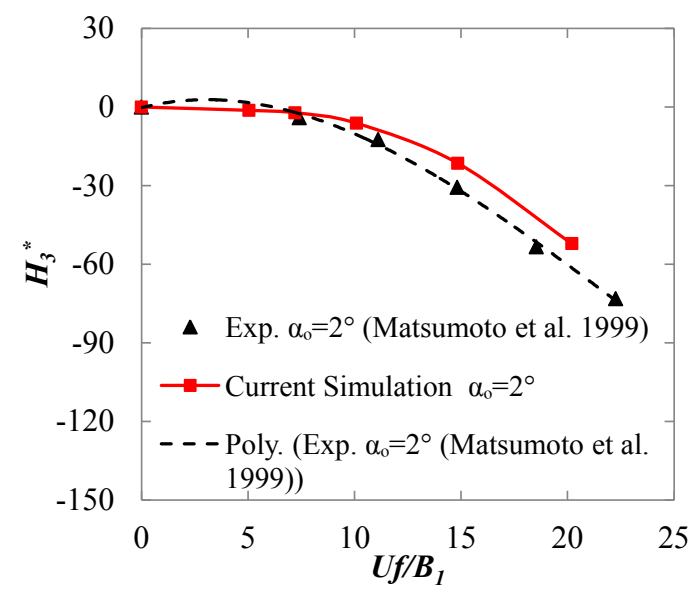

(b)

Fig. 5. Torsional $\left(A_{2}{ }^{*}\right.$ and $\left.H_{3}{ }^{*}\right)$ flutter derivatives of the pentagonal bridge deck at $R_{e B}$ of $6.0 \times 10^{4}$

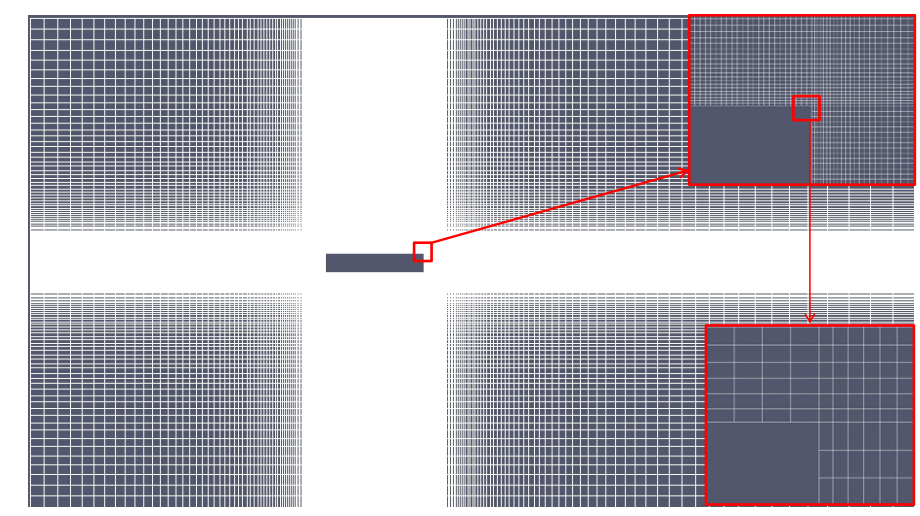

Fig. 6. Computational grid system for the rectangular cylinder (Side ratio of 5) 


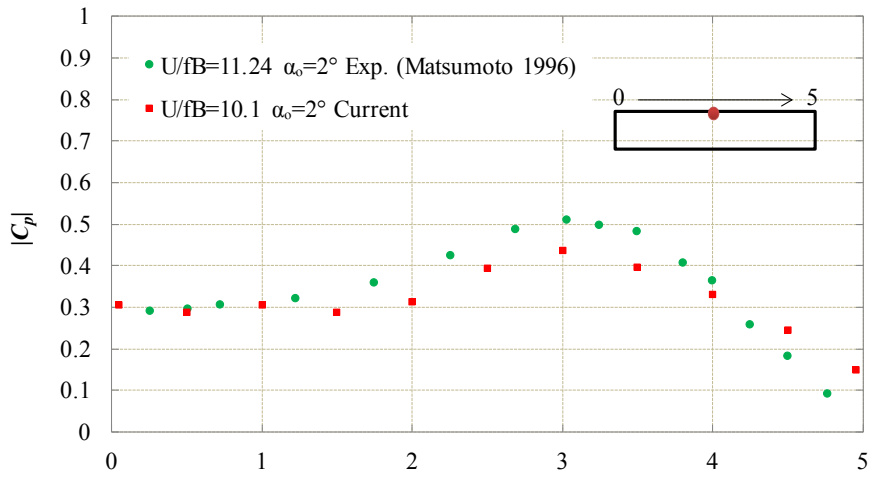

(a) Amplitude of unsteady pressure $\left(\left|C_{P}\right|\right)$

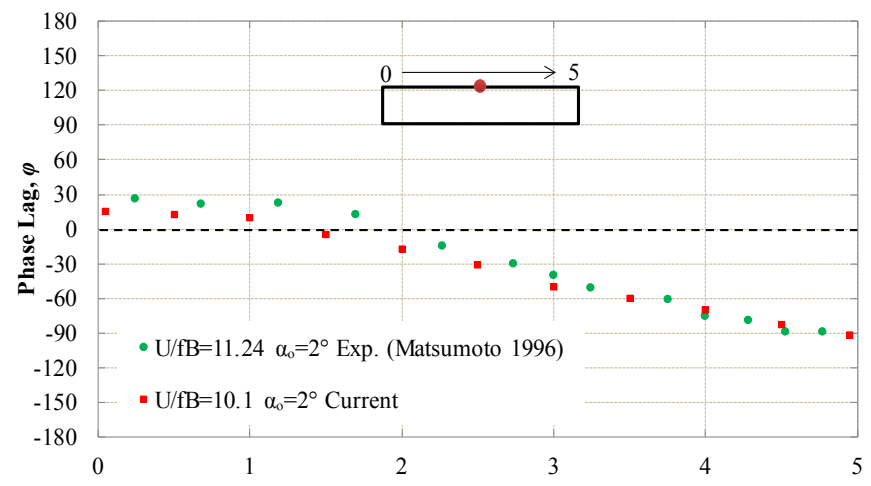

(b) Phase lag $(\varphi)$

Fig. 7. Unsteady pressure characteristics of the rectangular cylinder $(R=5)$ on top surface

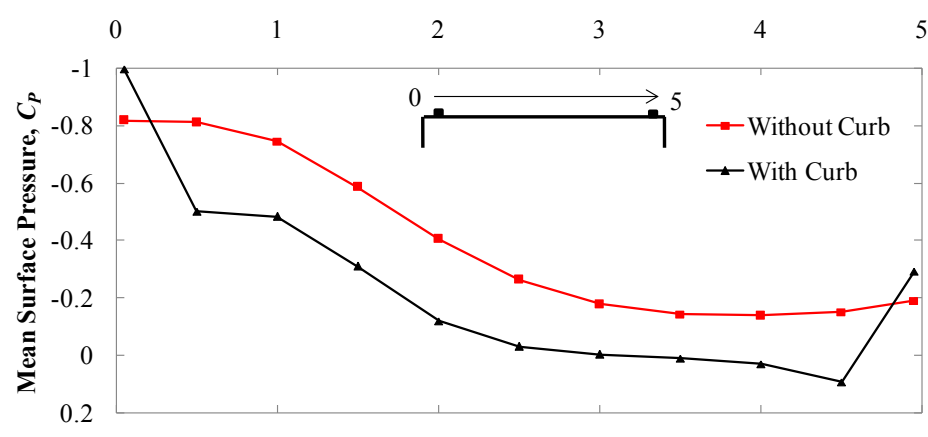

(a) Top Surface

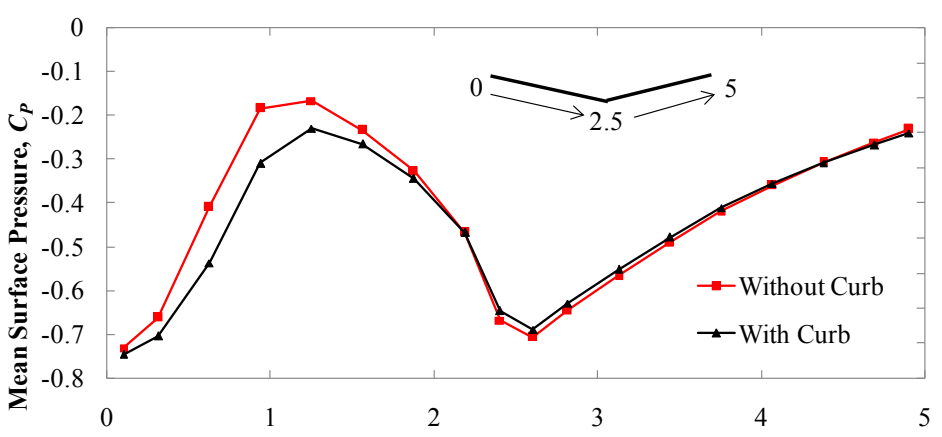

(b) Bottom Surface

Fig. 8. Mean value of surface pressure distribution around the decks 


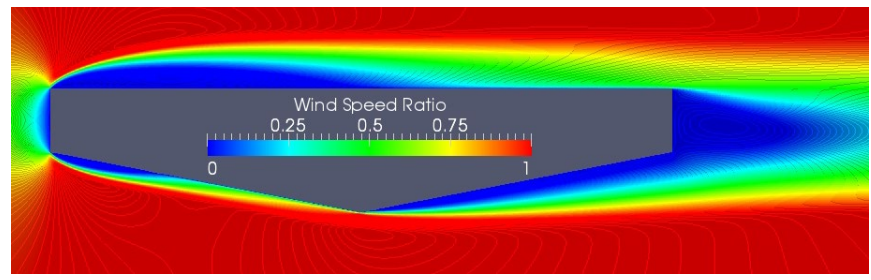

(a) Without Curb

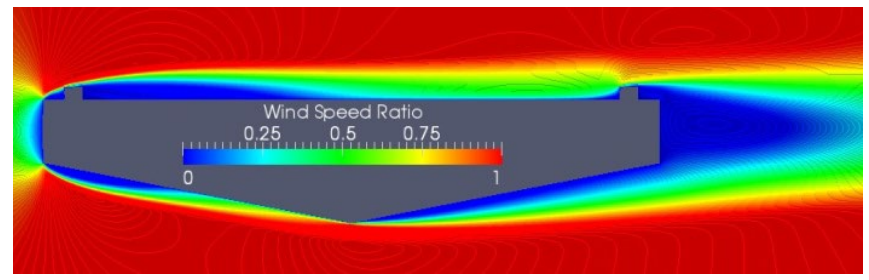

(a) With Curb

Fig. 9. Influence of SIM on wind speed ratio around the bridge decks

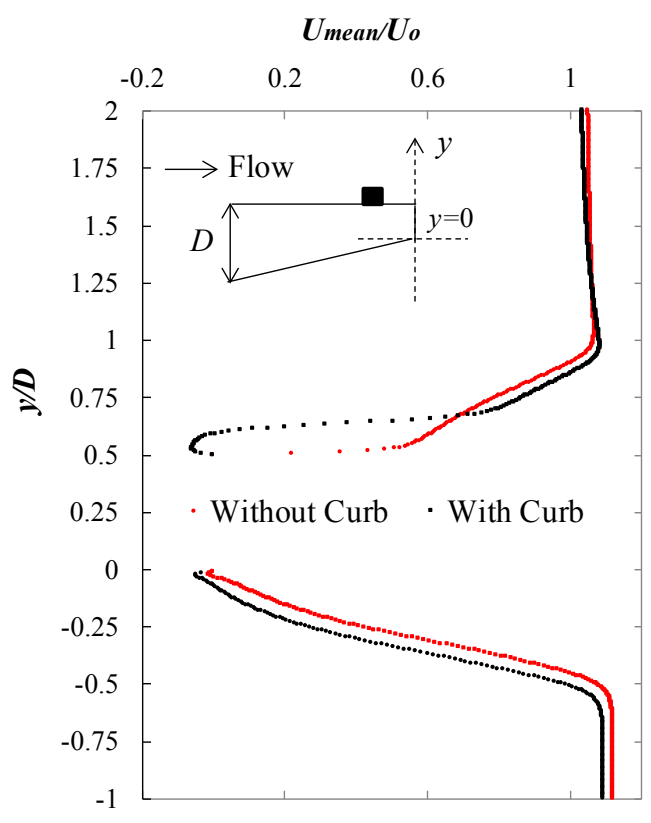

Fig. 10. Influence of SIM on trailing edge flow separation

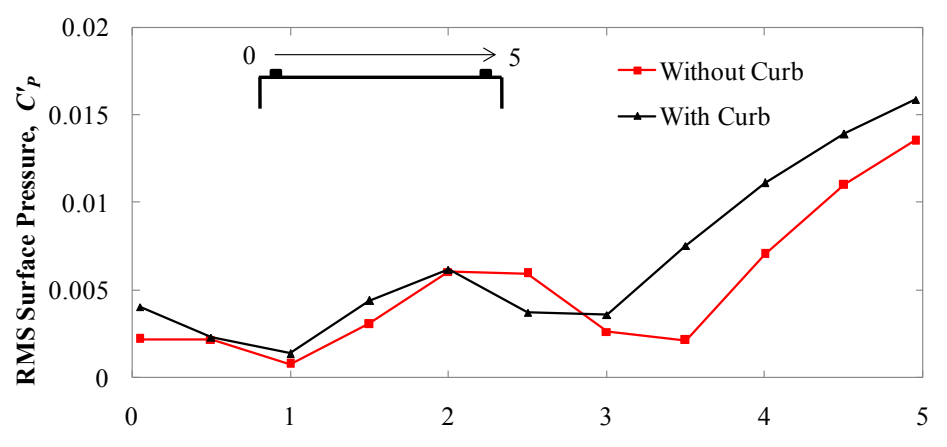

(a) Top Surface 


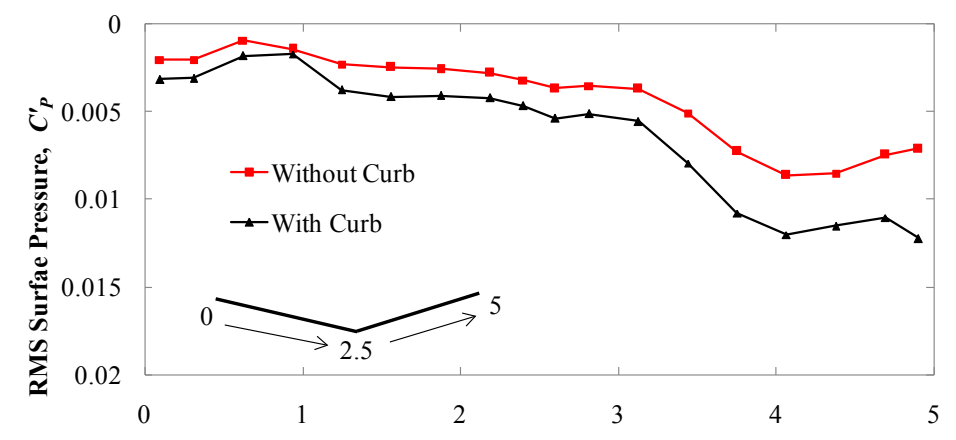

(b) Bottom Surface

Fig. 11. RMS Surface pressure distribution around the bridge decks
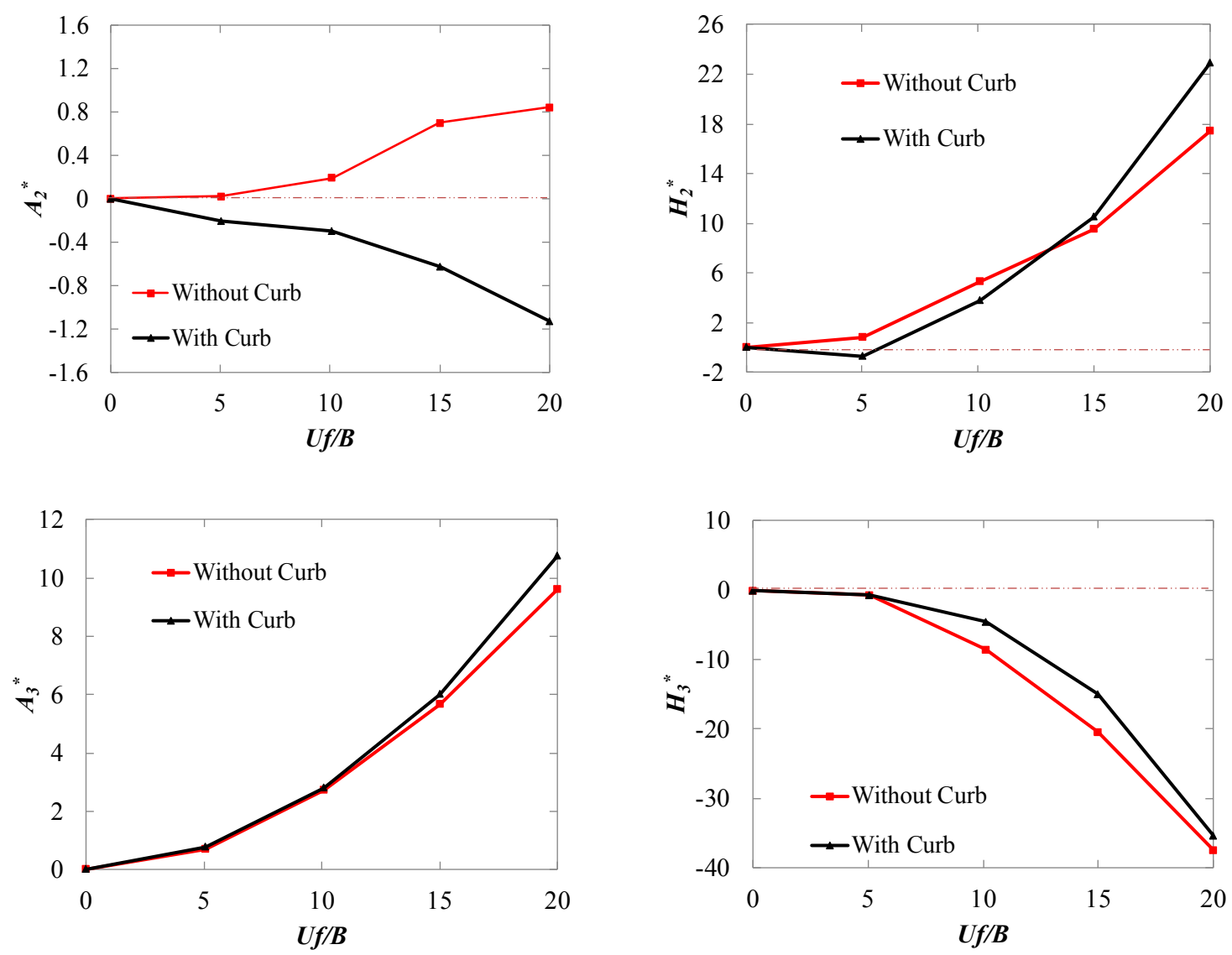

Fig. 12. Influence of SIM on torsional flutter derivatives 


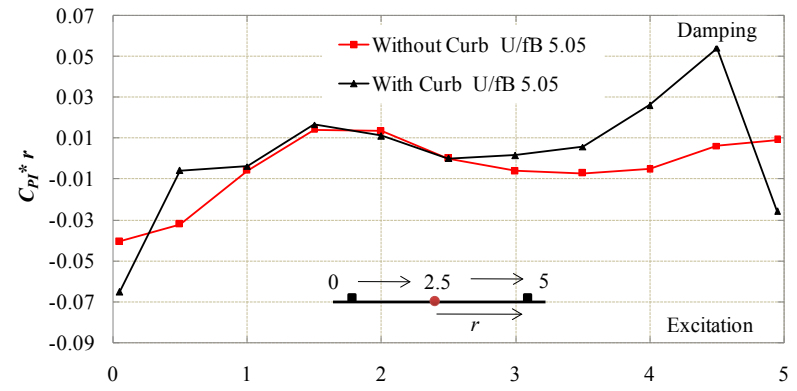

(a) Top Surface

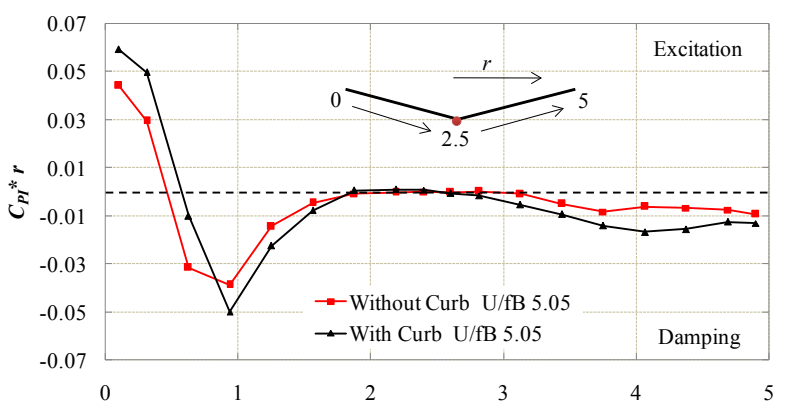

(b) Bottom Surface

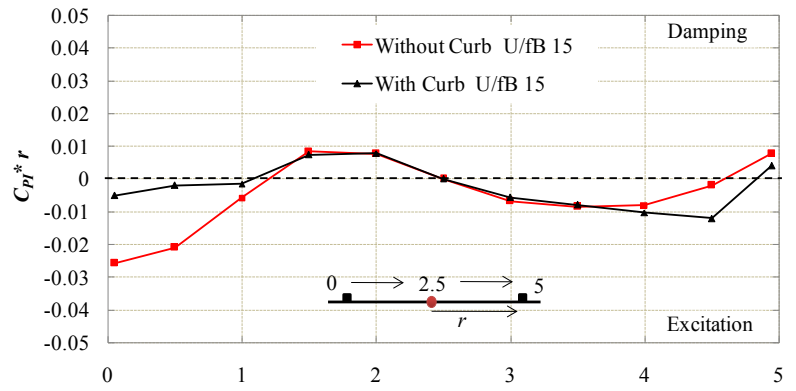

(c) Top Surface

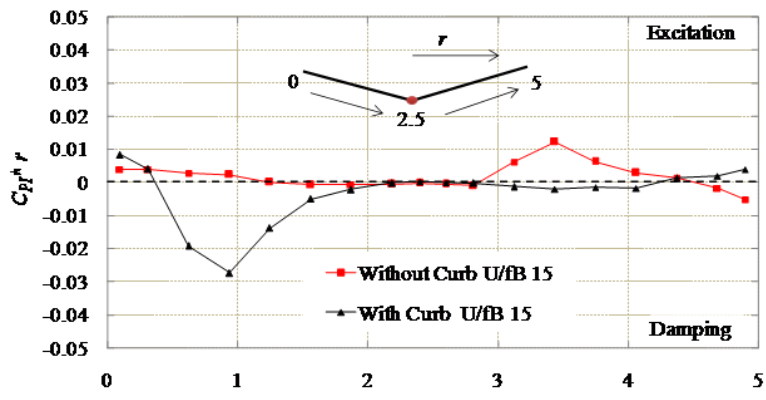

(d) Bottom Surface

Fig. 13. Influence of SIM on work done by the unsteady pressure

\section{References}

[1] Yoshida, K., Kubo, Y., Tsuji, E., Kimura, K. and Kato, K.: Effects of lower plate slope on the aerodynamic characteristics of a pentagonal cross section bridge deck. Proceedings of $19^{\text {th }}$ National Symposium on Wind Engineering, 295-300 (2006).

[2] Kubo, Y., Yoshida, K., Tuji, E., Kimura, K. and Kato, K.: Development of aerodynamically stable bridge girder cross section for long span bridges. In proceedings of $12^{\text {th }}$ Int. Conf. on Wind Engineering, 1-6 July, Cairns, Australia, 239-246 (2007).

[3] Noda, T., Kubo, Y., Kimura, K., Kato, K., Okubo, K. and Yoshida, K.: The effect of lower flange slope on the aerodynamic stability on a pentagonal cross-section girder. Journal of Civil Engineering (JSCE), 65(3), 797-807 (2009) (Japanese).

[4] Kubo, Y., Hayashida, K., Noda, T. and Kimura, K.: Mechanism on reduction of aerodynamic forces and suppression of aerodynamic response of a square prism due to separation interface method. In Proceedings of 6th Int. Colloq. On Bluff Body Aerodynamics and Applications, 20-24 July, Milano, Italy,1-4, (2008).

[5] Haque, M.N., Katsuchi, H., Yamada, H. and Nishio, M.: Flow field analysis of a pentagonal-shaped bridge deck by 
unsteady RANS. Engineering Application of Computational Fluid Mechanics, 10(1), 1-16(2015).

[6] Bruno, L. and Mancini, G.: Importance of deck details in bridge aerodynamics. Structural Engineering International, 12(4), 289-294 (2002).

[7] Mannini, C., Soda, A., Vob, R. and Schewe, G.: Unsteady RANS simulation of flow around a bridge section. Journal of Wind Engineering and Industrial Aerodynamics, 98, 742-753 (2010).

[8] Nieto, F., Kusano, I., Hernandez, S. and Jurado, J.A.: CFD analysis of the vortex-shedding response of twin-box deck cable-stayed bridge. In proceedings of $5^{\text {th }}$ Int. Symp. on Computational Wind Engineering, 23-27 May, North carolina, USA, 1-8 (2010).

[9] Patruno, L.: Accuracy of numerically evaluated flutter derivatives of bridge deck sections using RANS: Effects on the flutter onset velocity. Engineering Structures, 89, 49-65 (2015).

[10] Menter, F.R.: Two-equation eddy-viscosity turbulence models for engineering application. AIAA Journal 32(8), $1589-1605$ (1994)

[11] Menter, F.R., Kuntz, M. and Langtry, R. Ten years of industrial experience with the SST turbulence model. Turbulence, Heat and Mass Transfer, 4, 625-632 (2003).

[12] Haque, M.N., Katsuchi, H., Yamada, H. and Nishio, M.: Strategy to develop efficient grid system for flow analysis around two-dimensional bluff bodies. KSCE Journal of Civil Engineering, 20(05), 1-12 (2015).

[13] Ferziger, J.H. and Perić, M.: Computational Methods for Fluid Dynamics. Springer, Second Edition (2002).

[14] Donea, J., Huerta, A., Ponthot, J.Ph., Rodri'guez-Ferran, A.: Encyclopedia of Computational Mechanics. Volume 2 Solids and Structures.Chapter Arbitrary Lagrangian-Eulerian Methods.Wiley, Chichester, 413-438 (2004).

[15] Bos, F.: Numerical simulation of flapping foil and wind aerodynamics: Mesh de-formation using radial basis functions. PhD Thesis, Technical University Delft, the Netherland (2009).

[16] Simiu, E. and Scalan, R.H.: Wind Effects on Structures: Fundamentals and Applications to Design. Wiley publisher (1996).

[17] Larsen, A. and Walther, J.H.: Discrete vortex simulation of flow around five generic bridge deck sections. Journal of Wind Engineering and Industrial Aerodynamics, 77 \& 78, 591-602 (1998). 
[18] Šarkić, A., Fisch, R., Höffer, R. and Bletzinger, K.: Bridge flutter derivatives based on computed, validated pressure fields. Journal of Wind Engineering and Industrial Aerodynamics, 104-106, 141-151 (2012).

[19] Brusiani, F., De, Miranda, S., Patruno, L., Ubertini, F. and Vaona, P. On the evaluation of bridge deck flutter derivatives using RANS turbulence model. Journal of Wind Engineering and Industrial Aerodynamics, 119:39-47 (2013). [20] Miranda, S.D., Patruno, L., Ubertini, F. and Vairo,G.: On the identifications of the flutter derivatives of bridge deck via RANS turbulence models: Benchmarking on rectangular prisms. Engineering Structures, 76, 359-370 (2014).

[21] Nieto, F., Owen, J.S., Hargreaves, D.M. and Hernandez, S.: Bridge deck flutter derivatives: efficient numerical evaluation exploiting their independencies. Journal of Wind Engineering and Industrial Aerodynamics, 136, 138-150 (2015).

[22] Miyata, T., Miyazaki, M. and Yamada, H. Pressure distribution for wind induced vibrations of box girder bridges. Journal of Wind Engineering and Industrial Aerodynamics, 14, 223-234 (1983).

[23] Nagao, F., Utsunomiya, H., Yoshioka, E., Ikeuchi, A. and Kobayashi, H.: Effects of handrails on separated shear flow and vortex-induced oscillation. Journal of Wind Engineering and Industrial Aerodynamics, 69-71, 819-827 (1997).

[24] Matsumoto, M., Daito, Y., Yoshizumi, F., Ichikawa, Y. and Yabutani, T.: Torsional flutter of bluff bodies. Journal of Wind Engineering and Industrial Aerodynamics, 69-71, 871-882 (1997).

[25] Sukamta, Nagao, F., Noda, M. and Muneta, K.: Aerodynamic stabilizing mechanism of a cable stayed bridge with two edge box girder. In Proceedings of $6^{\text {th }}$ International Colloquium on Bluff Body Aerodynamics and Applications, Milano, Italy, 20-24 July, (2008).

[26] Shirai, S. and Ueda, T.: Aerodynamic simulation by cfd of flat box girder of super-long span suspension bridge. Journal of Wind Engineering and Industrial Aerodynamics, 91, 279-290 (2003).

[27] Matsumoto, M., Yoshizumi, F., Yabutani, T. and Nakajima, N.: Flutter stabilization and Heaving-branch flutter. Journal of Wind Engineering and Industrial Aerodynamics, 83 (1-3), 289-299 (1999).

[28] Sarwar, M.W., Ishihara, T., Shimada, K., Yamasaki, Y. and Ikeda, T.: Prediction of aerodynamic characteristics of a box girder bridge section using the LES turbulence model. Journal of Wind Engineering and Industrial Aerodynamics, 96, 1895-1911 (2008). 
[29] Matsumoto, M., Kobayashi, Y. and Shirato, H.: The influence of aerodynamic derivatives on flutter. Journal of Wind Engineering and Industrial Aerodynamics, 60, 227-239 (1996). 